\title{
Lymphomagenesis in Circulating Tumor Cell-Derived Xenograft Models
}

\author{
Dhwani Patel ${ }^{1}$, Youbin Zhang ${ }^{2}$, Madoka Kawaguchi ${ }^{1}$, Massimo Cristofanilli ${ }^{2}$, Huiping Liu ${ }^{1,3}$ and Xia \\ Liu*1 \\ ${ }^{1}$ Department of Pharmacology, Feinberg School of Medicine, Northwestern University, Chicago, IL 60611, USA \\ ${ }^{2}$ Department of Medicine, Hematology/Oncology Division, Northwestern University Feinberg School of Medicine, Chicago, IL 60611, \\ USA \\ ${ }^{3}$ Robert H. Lurie Comprehensive Cancer Center, Feinberg School of Medicine, Northwestern University, Chicago, IL 60611, USA \\ *Corresponding author: Xia Liu, Northwestern University Feinberg School of Medicine, Department of Pharmacology, Chicago, IL \\ 60611, USA, email: xia.liu@northwestern.edu
}

\section{ARTICLE INFO}



Published: May 15, 2019

\section{ABSTRACT}

Abbreviations: PDX: Patient Derived Xenograft; CTC: Circulating Tumor Cells; NSG: NOD Scid Gamma Mouse; CDXs: CTC-Derived Xenograft Models; EBV: Epstein-Barr Virus; RBCs: Red Blood Cells

Citation: Dhwani Patel, Youbin Zhang, Madoka Kawaguchi, Massimo Cristofanilli, Huiping Liu, Xia Liu. Lymphomagenesis in Circulating Tumor Cell-Derived Xenograft Models. Biomed J Sci \& Tech Res 18(1)-2019. BJSTR. MS.ID.003094.

\section{Short Communication}

Patient derived xenograft (PDX) models are useful model systems in cancer research to represent human cancer heterogeneity, intratumor and inter-tumor, and offer greater predictive value than traditional cell line-derived xenografts. They are indispensable, personalized models for preclinical drug screening, biomarker identification, molecular mechanistic studies, and personalized precision medicine. One of the most widely used approach to establish PDX tumor model is based on the transplantation of primary or metastatic human tumors (or pleural effusion) directly into highly immunodeficient mice, such as nonobese diabetic/ severe combined immunodeficient mice (NOD/Scid) and NOD scid gamma mouse (NSG), followed by continuous propagation of the established heterotopic or orthotopic engraftment in mice [1-3] However, these PDX models require invasive surgical procedure to collect fresh solid tumor, metastatic lesion or pleural effusions from a patient which is costly and exposes the patient to suffer the pain and increased risk of morbidity. Circulating tumor cells (CTCs) are tumor cells escape from the primary tumor and enter circulation. They are considered the seeds of metastasis, which account for more than $90 \%$ cancer-related deaths.
Recently, CTCs from prostate and lung cancer patients have been used to successfully generate prostate cancer and lung cancerderived PDX models respectively $[4,5]$. Comparing to conventional PDX models, CTC-derived xenograft models (CDXs) have many advantages including minimal pain/risk to patients and easily noninvasive obtained samples at any phase of the disease progression. However, there are several limitations and challenges to establish CDXs including the rare event of CTCs and specific devices required to isolate CTCs which are not available in every laboratory. Ours and other studies have demonstrated that CTC exist as both single cells and clusters, and CTC clusters have increased metastatic ability and stemness than single CTCs [6,7]. Therefore, we hypothesize that CTC clusters could have higher potential to engraft in mice to generate CTXs than single CTCs. The Cell Search TM system is the first and only clinically validated, FDA-cleared system for identification, isolation, and enumeration of CTCs of epithelial origin (CD45-, EpCAM+, and cytokeratins) in whole blood. In our ongoing project, we are making efforts to establish breast cancer CDXs.

To increase success rate, we first used CellSearchTM to select and process the samples from patients with CTC clusters (Figure 
1). In order to avoid losing CTCs during blood process, we directly orthotopically injected white blood cells (WBCs) into mammary fat pats of immunodeficient NSG mice after red blood cells (RBCs) lysis. Unexpected, all mice injected with these WBCs developed tumors expressing CD20 but not Ep CAM, indicating these tumors are lymphoma but not breast cancer (Figure 2). Therefore, we modified our protocol to deplete CD45 positive mononuclear cells either by BD cell sorter or MACS CD45 microbeads to enrich CTCs from peripheral blood samples collected from metastatic breast cancer patients with CTC clusters. The enriched CTCs are then injected into
NSG mice. By this approach, we have successfully generated breast cancer CDXs from blood samples with 200-1000 CTCs $/ 7.5 \mathrm{ml}$ blood counted by Cell Search. We then searched for the reasons why lymphoma developed in these NSG mice. We found that the development of lymphoma from human tumor sample xenografts is not a new phenomenon. Several studies have revealed that human tumors engrafted in immunodeficient mice are susceptible to Epstein-Barr virus (EBV)-associated lymphoma-genesis during generation of conventional PDXs [8-10].
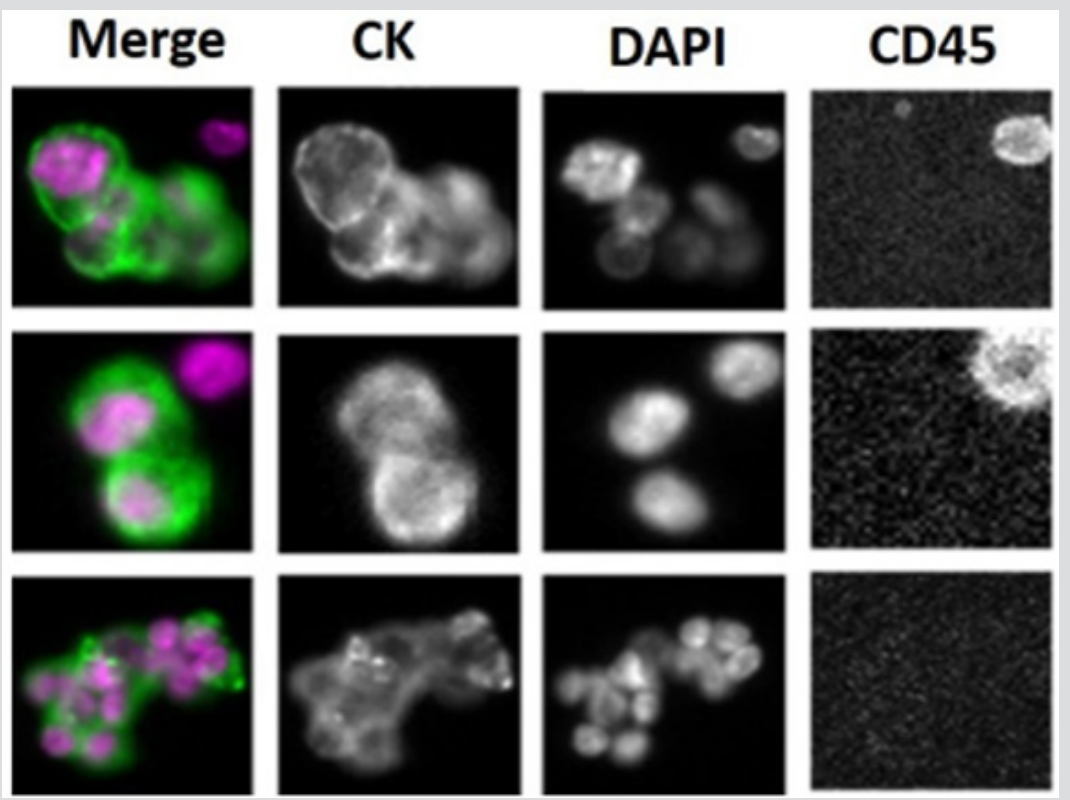

Figure 1: Representative images of CTCs clusters from metastatic breast cancer patients captured by CellSearch platform. AntiCK: epithelial marker to identify breast cancer cells: DAPI: to stain nucleus; anti- CD45: to stain blood cells.
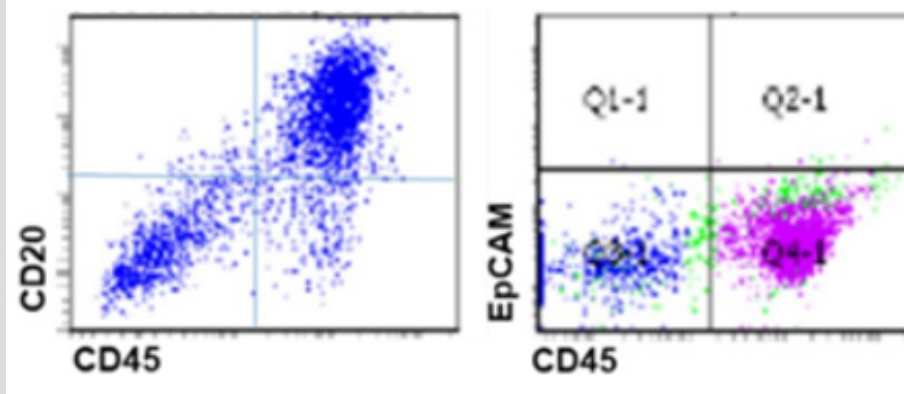

\section{Lymphoma}

(without CD45 depletion)
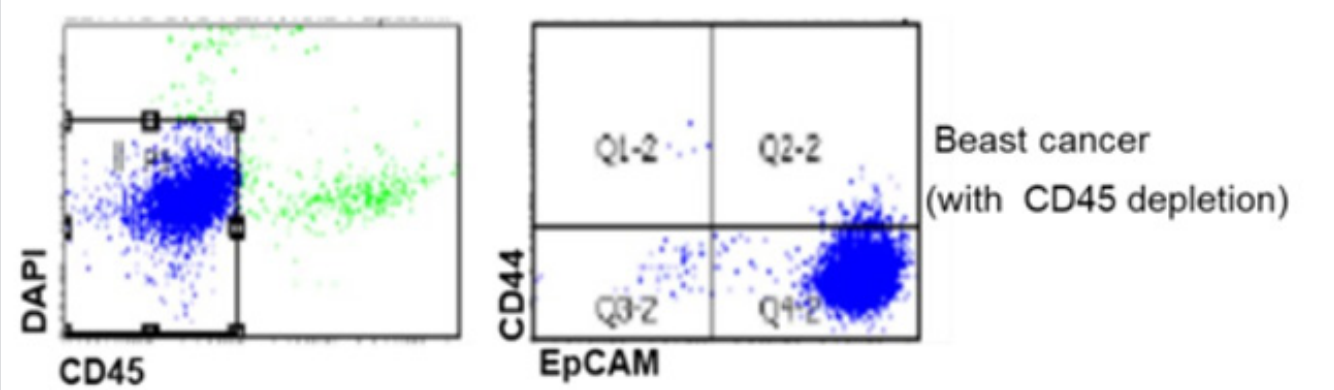

Figure 2: Characterization of established CDXs by flow cytometry. Upper panel shows NSG mouse developed CD20+ lymphoma from WBC blood sample without CD45 depletion. Lower panel shows EpCAM+ breast cancer CTXs established from CD45 depleted blood sample. 
EBV infects over $90 \%$ of humans persisting for the lifetime of immunocompetent individuals without symptom. They can infect human B-cells, T-cells, NK cells, and epithelial cells. Under immunocompromised condition, these latent infected cells can develop lymphomas. Because B-cells are typically present in solid tumors, the development of lymphoma is a common problem during generation of conventional PDXs with the range from $17 \%$ (in SCID mice) to $80 \%$ in NSG mice [10]. Although CDX models have great potential in the development of personalized therapy for cancer patients, the lymphomagenesis during generation of CDXs suggest that careful screening of lymphocytic markers ( such as CD20,CD19) are necessary to ensure the accuracy of generated CDXs. It is worth mentioning that we noticed that it takes shorter time to develop lymphoma (2-3 months) than breast cancer (5-8 months). Therefore, the faster the tumor forms, the higher chance the mice develop lymphoma instead of expected tumor.

\section{Acknowledgement}

This manuscript has been partially supported by Abbvie-NU Collaboration in Oncology Grant (X.L).

\section{References}

1. Hidalgo M, F Amant, AV Biankin, E Budinska, AT Byrne, et al. (2014) Patient-derived xenograft models: an emerging platform for translational cancer research. Cancer Discov 4(9): 998-1013.

2. Siolas D, GJ Hannon (2013) Patient-Derived Tumor Xenografts: Transforming Clinical Samples into Mouse Models. Cancer Research 73(17): 5315-5319.

\section{ISSN: 2574-1241}

DOI: 10.26717/BJSTR.2019.18.003094

Xia Liu. Biomed J Sci \& Tech Res

This work is licensed under Creative Commons Attribution 4.0 License

Submission Link: https://biomedres.us/submit-manuscript.php
3. Liu H, MR Patel, JA Prescher, A Patsialou, D Qian, et al. (2010) Cancer stem cells from human breast tumors are involved in spontaneous metastases in orthotopic mouse models. Proc Natl Acad Sci U S A 107(42): 1811518120.

4. Williams ES, V Rodriguez Bravo, U Chippada Venkata J De Ia Iglesia Vicente, Y Gong, et al. (2015) Generation of Prostate Cancer Patient Derived Xenograft Models from Circulating Tumor Cells. J Vis Exp 105: 53182.

5. Hodgkinson CL, CJ Morrow, Y Li, RL Metcalf, DG Rothwell, et al. (2014) Tumorigenicity and genetic profiling of circulating tumor cells in smallcell lung cancer. Nat Med 20(8): 897-903.

6. Liu X, R Taftaf, M Kawaguchi, YF Chang, W Chen, et al. (2019) Homophilic CD44 Interactions Mediate Tumor Cell Aggregation and Polyclonal Metastasis in Patient-Derived Breast Cancer Models. Cancer Discov 9(1): 96-113.

7. Aceto N, A Bardia, DT Miyamoto, MC Donaldson, BS Wittner, et al. (2014) Circulating tumor cell clusters are oligoclonal precursors of breast cancer metastasis. Cell 158(5): 1110-1122.

8. Chen K, S Ahmed, O Adeyi, JE Dick, A Ghanekar (2012) Human solid tumor xenografts in immunodeficient mice are vulnerable to lymphomagenesis associated with Epstein-Barr virus. PLoS One 7(6): e39294.

9. Bondarenko G, A Ugolkov, S Rohan, P Kulesza, O Dubrovskyi, et al. (2015) Patient-Derived Tumor Xenografts Are Susceptible to Formation of Human Lymphocytic Tumors. Neoplasia 17(9): 735-741.

10. Taurozzi AJ, R Beekharry, M Wantoch, MC Labarthe, HF Walker, et al. (2017) Spontaneous development of Epstein-Barr Virus associated human lymphomas in a prostate cancer xenograft program. PLoS One 12: e0188228.

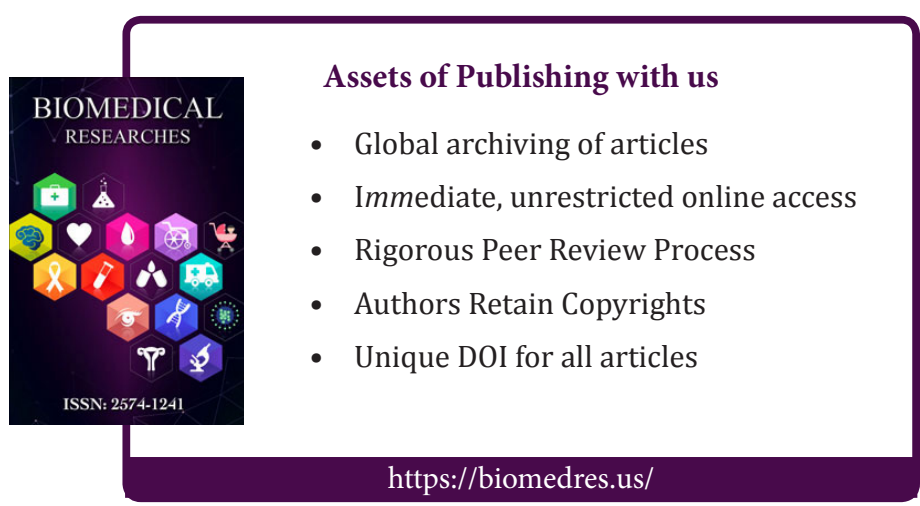

